\title{
Spontaneous Retroperitoneal Hematoma: a Case Report, Review of Literature, and Proposal of Diagnostic and Management Algorithm
}

\section{Takuma Kurotaki}

Teine Keijinkai Hospital: Teine Keijinkai Byoin https://orcid.org/0000-0002-6134-9156

Naoya Okada ( $\sim$ okada-na@keijinkai.or.jp )

Department of Surgery, Teine Keijinkai Hospital https://orcid.org/0000-0002-1443-9191

\section{Yasuo Sakurai}

Teine Keijinkai Hospital: Teine Keijinkai Byoin

\section{Takumi Yamabuki}

Teine Keijinkai Hospital: Teine Keijinkai Byoin

\section{Minoru Takada}

Teine Keijinkai Hospital: Teine Keijinkai Byoin

\section{Kentaro Kato}

Teine Keijinkai Hospital: Teine Keijinkai Byoin

\section{Takeshi Yokoyama}

Teine Keijinkai Hospital: Teine Keijinkai Byoin

\section{Yoshiyasu Ambo}

Teine Keijinkai Hospital: Teine Keijinkai Byoin

Yoshihiro Kinoshita

Teine Keijinkai Hospital: Teine Keijinkai Byoin

\section{Fumitaka Nakamura}

Teine Keijinkai Hospital: Teine Keijinkai Byoin Nobuichi Kashimura

Teine Keijinkai Hospital: Teine Keijinkai Byoin

\section{Case report}

Keywords: spontaneous retroperitoneal hematoma, conservative management, transcatheter arterial embolization, surgical intervention, algorithm

Posted Date: October 18th, 2021

DOl: https://doi.org/10.21203/rs.3.rs-958926/v1 
License: (c) (i) This work is licensed under a Creative Commons Attribution 4.0 International License. Read Full License 


\section{Abstract}

Background: Spontaneous retroperitoneal hematoma ( $\mathrm{SRH})$ is defined as bleeding in the retroperitoneal space without any triggers such as trauma, invasive procedures, and abdominal aortic aneurysm.

Case presentation: A 48-year-old man who experienced sudden abdominal pain, severe hypotension, and decreased hemoglobin $(\mathrm{Hb})$ was diagnosed with $\mathrm{SRH}$. Contrast-enhanced computed tomography (CT) revealed massive left retroperitoneal hematoma; however, neither extravasation nor causative aneurysm was noted. Through conservative management with close monitoring, he was treated and discharged on the $10^{\text {th }}$ hospital day without any morbidity.

Conclusions: SRH treatment comprises conservative management, transcatheter arterial embolization, and surgical intervention. The mortality rate of $\mathrm{SRH}$ is so high that the optimal treatment timing needs to be carefully judged based on detailed evaluation and management algorithm with clear criteria.

\section{Background}

Spontaneous retroperitoneal hematoma $(\mathrm{SRH})$ is a potentially fatal disease that usually occurs during antiplatelet or anticoagulant therapy [1]. The treatment comprises conservative management, transcatheter arterial embolization (TAE), and surgical intervention [2]. These treatment options are selected case-by-case without guidelines. We report a case of SRH resulting in hemorrhagic shock, which was successfully treated via conservative management with close monitoring, and propose a diagnostic and management algorithm based on our experience and a review of the existing literature.

\section{Case Presentation}

A 48-year-old man was admitted to a local hospital with sudden abdominal pain. Upon arrival, his systolic blood pressure was $50 \mathrm{mmHg}$, and he started receiving dobutamine. His medical history was significant for hypertension and dyslipidemia. Computed tomography (CT) revealed a low-density area in the left upper quadrant, and gastrointestinal stromal tumor (GIST) was suspected. His hemoglobin ( $\mathrm{Hb}$ ) level was $14.2 \mathrm{~g} / \mathrm{dL}$ on arrival, which decreased to $8.7 \mathrm{~g} / \mathrm{dL}$ on the following day; hence, he was referred to us for further examination and treatment. Upon arrival at our hospital, his circulation was maintained by continuous infusion of $7 \mu \mathrm{g} / \mathrm{kg} / \mathrm{min}$ of dobutamine. Contrast-enhanced CT revealed a massive homogeneous low-density area in the left retroperitoneum, which was suspected to be a giant hematoma (Figure 1a, b). No extravasation, arterial aneurysm, nor tumor was observed. An arterial line and a central venous catheter were introduced, and dobutamine was switched to noradrenaline. He had renal dysfunction (eGFR: $19 \mathrm{ml} / \mathrm{min} / 1.7$ ), which was possibly due to severe dehydration and compression of the renal parenchyma and renal vessels. He did not take any anticoagulant or antiplatelet medications and his coagulation status was normal. Angiography was not performed because the contrast-enhanced CT did not reveal extravasation and he had severe renal dysfunction. Because his circulation was maintained with fluid resuscitation, noradrenaline, and blood transfusion, conservative treatment with 
close monitoring in the intensive care unit (ICU) was indicated. Noradrenaline administration was terminated on the next day. A total of 8 units of red blood cells were administered to maintain his Hb level within 7-9 $\mathrm{g} / \mathrm{dL}$. He was discharged from the ICU on the 5th day because his symptoms had resolved and the contrast-enhanced CT did not reveal hematoma growth (Figure 2a). On the 7th day, enhanced magnetic resonance image (MRI) revealed heterogenous low T1 and high T2 signals (Figure 1c, d, e, f); therefore, hematoma was confirmed. No causative bleeding tumor was observed. As his vital signs were stable, we stopped monitoring them on the same day. He was discharged on the 10th hospital day without any morbidity. In the outpatient clinic, contrast-enhanced CT performed 5 months later revealed that the hematoma was remarkably reduced (Figure 2b). MRI performed at the same time revealed that the hematoma was localized to the retroperitoneal space, without the involvement of any other organs, suggesting that the source of the bleeding was not GIST (Additional File 1).

\section{Discussion And Conclusions}

$\mathrm{SRH}$ is a relatively rare clinical entity that involves bleeding in the retroperitoneal space without any triggers. SRH usually occurs in the population taking anticoagulants [1]. However, our patient was not taking any anticoagulant or antiplatelet medications. Bleeding can occur in any retroperitoneal vessels and organs [2]. Although the pathogenesis of SRH is unclear, it may be caused by arteriosclerosis of microvessels [2], vascular lesions like segmental arterial mediolysis [3], or unrecognized minor [2] trauma. The cause of bleeding is often unclear.

CT is a rapid and readily available test to identify retroperitoneal hematoma [4]. Extravasation is an independent predictor for TAE or surgical intervention [5]. MRI is helpful in establishing an accurate diagnosis owing to its efficient detection of retroperitoneal structures, including fat, organs, tumors, and hematoma [6]. Although MRI is not always necessary for diagnosis in the acute phase, it may be useful for differential diagnoses. Angiography is effective for detecting the hemorrhagic site and can enable the embolization of the affected arteries to stop the bleeding [7]. In the present case, contrast-enhanced CT revealed a low-density area in the central and left retroperitoneal space; however, neither extravasation nor causative aneurysm was observed. Enhanced MRI revealed heterogenous low T1 and high T2 signals indicating hematoma. Hence, the patient was diagnosed with $\mathrm{SRH}$.

We present the diagnostic and management algorithm of SRH based on a literature review (Figure 3). If the bleeding occurs because of aortic aneurysm rupture, urgent endovascular aneurysm repair (EVAR) or open surgical repair is required [8]. Retroperitoneal hematoma can be treated conservatively when there is no persistent bleeding and the circulation is maintained, whereas endovascular or surgical treatment is required when the circulation is unstable [2]. When the circulation is stable with continuous transfusion or vasopressor infusion, ongoing bleeding is suspected [9], and dynamic enhanced CT should be performed again.

Most patients with retroperitoneal hematoma take anticoagulant and/or antiplatelet medications.

Discontinuation of these antithrombotic agents and the reversal agents, such as vitamin $\mathrm{K}$, idarucizumab, 
and prothrombin complex concentrate, are recommended [10]. In the presence of congenital or acquired hemophilia, active prothrombin complex concentrate and recombinant factor Vlla should be administered [11]. The European guideline on the management of major bleeding and coagulopathy following trauma states that in cases of hemorrhagic shock, fluid resuscitation should be started and vasopressors, including norepinephrine, can be administered to achieve systolic blood pressure of $80-90 \mathrm{mmHg}[10]$. Transfusion is required to maintain $\mathrm{Hb}$ level of $7-9 \mathrm{~g} / \mathrm{dL}$ and platelet count above $50 \times 10^{9} / \mathrm{L}[10]$. The guideline also recommends treatment with fibrinogen concentrate or cryoprecipitate when Clauss fibrinogen level are $\leq 1.5 \mathrm{~g} / \mathrm{L}$ [10]. Fresh frozen plasma (FFP) administration is suggested if prothrombin time and/or activated partial thromboplastin time are $>1.5$ times the normal values [10]. In this case, the patient was in hemorrhagic shock, and the bleeding seemed to be persistent; however, his blood pressure could be maintained by fluid resuscitation, vasopressor infusion, and blood transfusion. Enhanced CT did not show extravasation and the bleeding site. Angiography was not performed because the possibility of active bleeding was less likely and embolization of the causative arteries was not necessary.

Conservative management with close monitoring in the ICU was chosen so that urgent TAE or laparotomy could be performed in case of prolonged and worsening shock state resulting from rebleeding. The retroperitoneal cavity is a relatively narrow space, and the tamponade effect of the hematoma reportedly stops bleeding [2]. Sunga et al. reported that the overall mortality of SRH within 30 days was as high as $10.1 \%$ [1]. Because of the high mortality rate, SRH cases need to be monitored carefully.

Tani et al. reported that in a retrospective study of 20 consecutive cases of SRH, TAE could achieve hemostasis in 17 out of 19 patients [7]. In four of these patients, the causative arteries could not be identified, and the arteries around the hematoma were embolized empirically [7]. Even if the origin of bleeding is not detected, empirical embolization might stop life-threatening bleeding. In this case, angiography was not performed because the patient was hemodynamically stable following fluid resuscitation and vasopressor infusion and had significant renal dysfunction due to dehydration.

Surgical treatment is performed to stop the bleeding and remove the hematoma [2]. Emergency laparotomy is indicated in cases of abdominal compartment syndrome and compression of the lumbar plexus or femoral nerve [12]. In a report of cases in which surgery was performed prior to TAE, the bleeding site was not intraoperatively identified in all of the four patients [13]. If the bleeding site cannot be identified, intra-abdominal packing can stop the retroperitoneal bleeding, and reoperation can be planned after 48 hours [10] for re-examining whether hemostasis is achieved [2]. In the hemodynamically unstable emergency cases, TAE should be performed in a hybrid operating room, and if the bleeding cannot be stopped by TAE, emergency laparotomy is needed.

We report a rare case of $\mathrm{SRH}$ with unstable hemodynamic status that was successfully managed via conservative treatment with close monitoring. As the mortality rate of $\mathrm{SRH}$ is high, $\mathrm{SRH}$ management requires systematic and close monitoring based on our suggested clear evaluation criteria and management algorithm.

\section{Abbreviations}


$\mathrm{SRH}=$ spontaneous retroperitoneal hematoma, $\mathrm{TAE}=$ transcatheter arterial embolization, $\mathrm{CT}=$ computed tomography, GIST = gastrointestinal stromal tumor, $\mathrm{Hb}=$ hemoglobin, ICU = intensive care unit, MRI = magnetic resonance image, EVAR = endovascular aneurysm repair, FFP = fresh frozen plasma

\section{Declarations}

\section{Ethics approval and consent to participate}

Ethics approval and consent to participate were waived because this study is a review of literature with a retrospective case report on a patient that provided consent for publication.

\section{Consent for publication}

Written informed consent was obtained from the patient for the publication of this case report and accompanying images.

\section{Availability of data and materials}

Not applicable.

\section{Competing interests}

The authors declare that they have no competing interests.

\section{Funding}

All authors declare that they received no funding.

\section{Authors' contributions}

All authors were involved in patient care, manuscript preparation, and literature search. Additionally, all authors read and approved the final manuscript.

\section{Acknowledgments}

We thank Edanz (https://jp.edanz.com/ac) for editing a draft of this manuscript.

\section{References}

1. Sunga KL, Bellolio MF, Gilmore RM, Cabrera D. Spontaneous retroperitoneal hematoma: etiology, characteristics, management, and outcome. J Emerg Med. 2012;43(2):e157-61.

2. Chan YC, Morales JP, Reidy JF, Taylor PR. Management of spontaneous and iatrogenic retroperitoneal haemorrhage: conservative management, endovascular intervention or open surgery? Int J Clin Pract. 2007;62(10):1604-13. 
3. Phillips CK, Lepor H. Spontaneous retroperitoneal hemorrhage caused by segmental arterial mediolysis. Rev Urol. 2006;8(1):36-40.

4. Caleo O, Bocchini G, Paoletta S, lerardi AM, Scionti A, Tonerini M, et al. Spontaneous non-aortic retroperitoneal hemorrhage: etiology, imaging characterization and impact of MDCT on management. A multicentric study. Radiol Medica. 2015;120(1):133-48.

5. Baekgaard JS, Eskesen TG, Lee JM, Yeh DD, Kaafarani HMA, Fagenholz PJ, et al. Spontaneous retroperitoneal and rectus sheath hemorrhage-management, risk factors and outcomes. World $\mathrm{J}$ Surg. 2019;43(8):1890-7.

6. Nishimura H, Zhang Y, Ohkuma K, Uchida M, Hayabuchi N, Sun S. MR imaging of soft-tissue masses of the extraperitoneal spaces. Radiographics. 2001 Sep 1;21(5):1141-54.

7. Tani R, Sofue K, Sugimoto K, Katayama N, Hamada MAS, Maruyama K, et al. The utility of transarterial embolization and computed tomography for life-threatening spontaneous retroperitoneal hemorrhage. Jpn J Radiol. 2019;37(4):328-35.

8. Wanhainen A, Verzini F, Van Herzeele I, Allaire E, Bown M, Cohnert T, et al. European Society for Vascular Surgery (ESVS) 2019 clinical practice guidelines on the management of abdominal aortoiliac artery aneurysms. Eur J Vasc Endovasc Surg. 2018

9. Brown CVR, Velmahos GC, Neville AL, Rhee P, Salim A, Sangthong B, et al. Hemodynamically "stable" patients with peritonitis after penetrating abdominal trauma: identifying those who are bleeding. Arch Surg. 2005;140(8):767-72.

10. Spahn DR, Bouillon B, Cerny V, Duranteau J, Filipescu D, Hunt BJ, et al. The European guideline on management of major bleeding and coagulopathy following trauma: fifth edition. Crit Care. 2019;23(1):98.

11. Berntorp E, Shapiro AD. Modern haemophilia care. Lancet. 2012;379(9824):1447-56.

12. Ekici Y, Aydogan C, Yagmurdur MC, Kirnap M, Harman A, Moray G, et al. Treatment approaches for spontaneous retroperitoneal bleeding. Int Surg 2009;94(2):171-5.

13. Isokangas JM, Perälä JM. Endovascular embolization of spontaneous retroperitoneal hemorrhage secondary to anticoagulant treatment. Cardiovasc Intervent Radiol. 2004;27(6):607-11.

\section{Table}

Due to technical limitations, table $1 \mathrm{xlsx}$ is only available as a download in the Supplemental Files section.

\section{Figures}




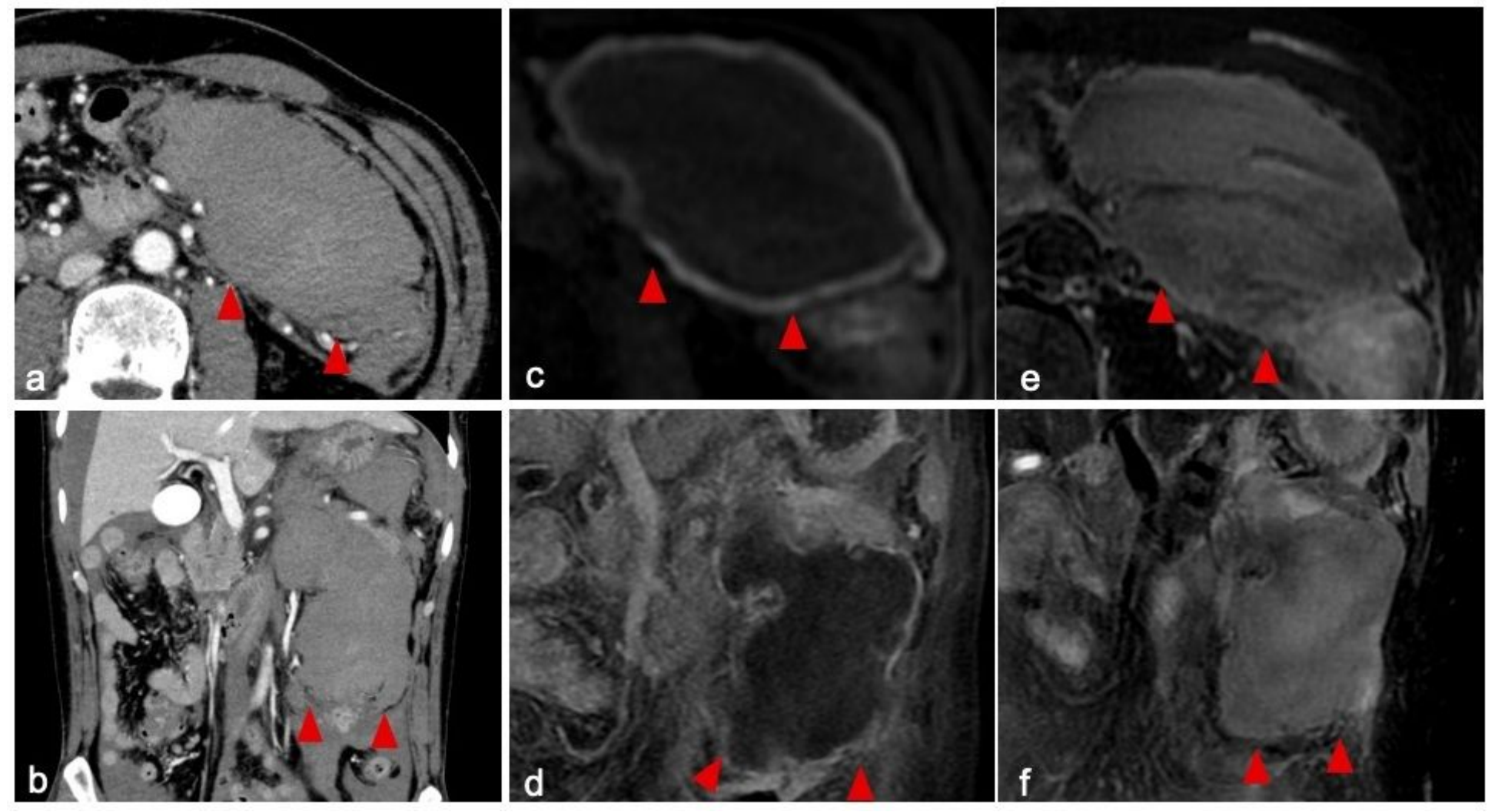

Figure 1

CT and MRI of the abdomen a, Contrast-enhanced CT revealed a large low-density area (arrow heads) in the left peritoneal space. b, Coronal contrast-enhanced CT revealed a low-density area (arrow heads) in the left retroperitoneal space. Neither extravasation nor causative aneurysm was observed. c, T1weighted MRI revealed that the mass (arrow heads) was encapsulated by hyperintense rims. $d$, Coronal T1-weighted MRI performed after gadolinium administration revealed no extravasation in the mass (arrow heads). e, T2-weighted MRI showed homogeneously high intensity in the mass (arrow heads). $\mathrm{f}$, Coronal T2-weighted MRI showed homogeneously high intensity in the mass (arrow heads). CT, computed tomography; MRI, magnetic resonance imaging 


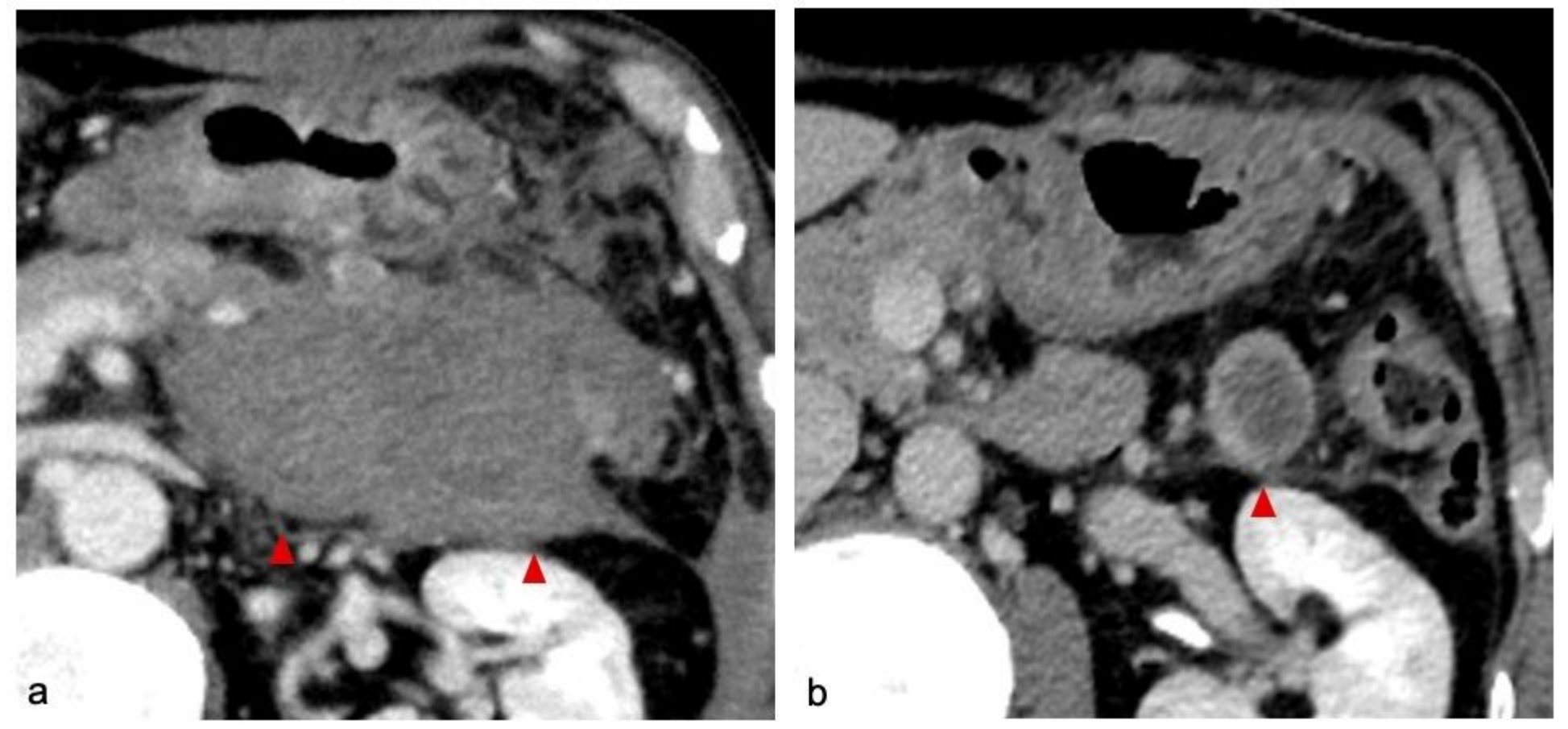

Figure 2

Contrast-enhanced CT of the abdomen a, Contrast-enhanced CT on the 5th hospital day showed a large hematoma (arrow heads) in the left peritoneal space. b, Contrast-enhanced CT 5 months after discharge revealed that the hematoma (arrow head) was remarkably reduced. CT, computed tomography

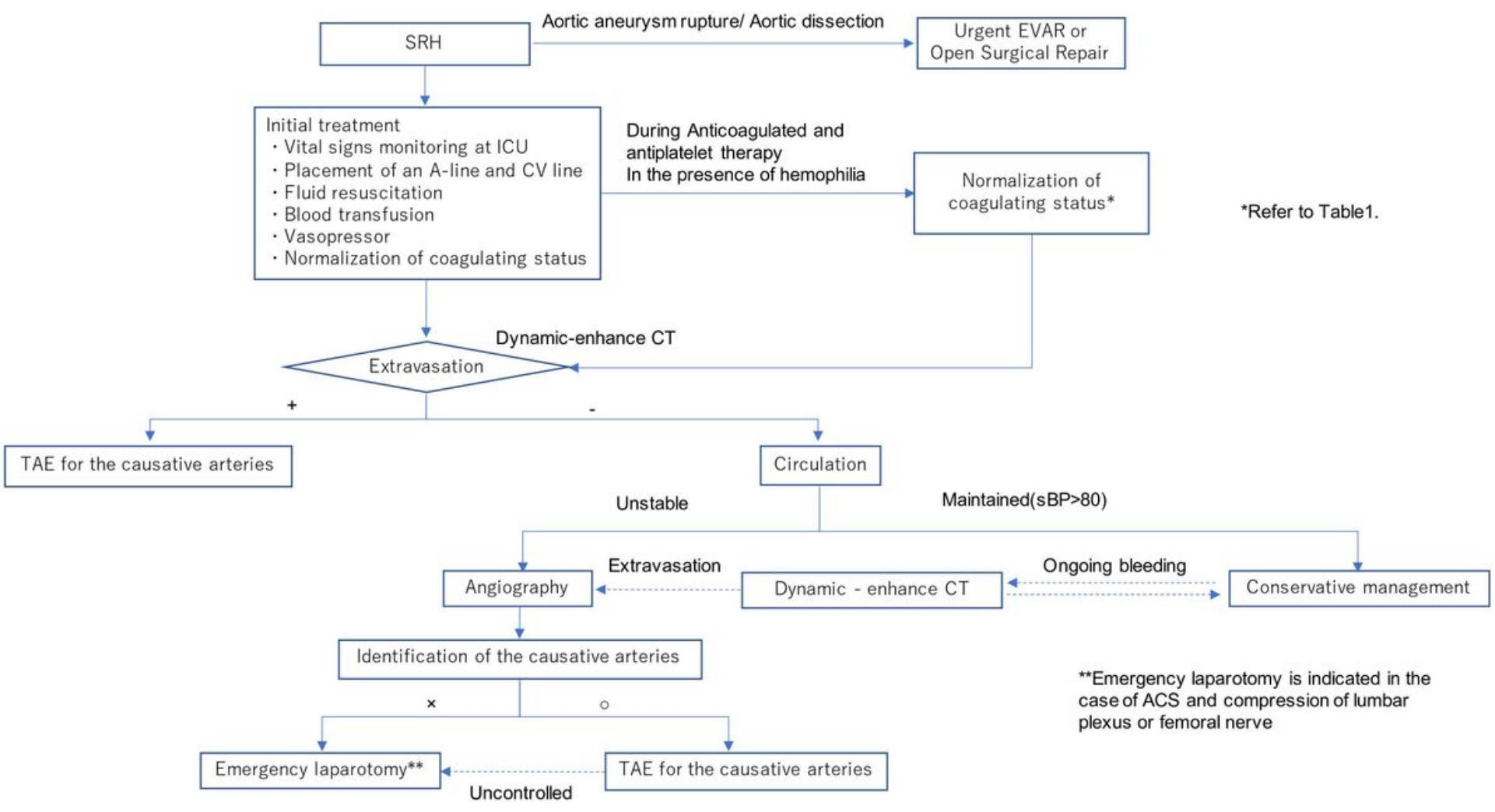

Figure 3 
Algorithm for diagnosis and treatment of retroperitoneal hematoma SRH, spontaneous retroperitoneal hematoma; EVAR, endovascular aortic repair; TAE, transcatheter arterial embolization; ACS, abdominal compartment syndrome

\section{Supplementary Files}

This is a list of supplementary files associated with this preprint. Click to download.

- Table1.xlsx

- Additionalfile1.jpg 